\title{
Clinical factors associated with arrhythmia and short-term prognosis following mitral valve repair: a retrospective cohort study
}

\author{
Yuxiang Zhu, Wenbin Jing, Pengfei Lv, Yubao Zhu, Zhigang Liu \\ Department of Cardiovascular Surgery, TEDA International Cardiovascular Hospital, Tianjin, China \\ Contributions: (I) Conception and design: Yuxiang Zhu, Z Liu; (II) Administrative support: Z Liu; (III) Provision of study materials or patients: \\ Yuxiang Zhu, W Jing, P Lv, Yubao Zhu; (IV) Collection and assembly of data: Yuxiang Zhu, W Jing, P Lv, Yubao Zhu; (V) Data analysis and \\ interpretation: Yuxiang Zhu, W Jing, P Lv, Yubao Zhu; (VI) Manuscript writing: All authors; (VII) Final approval of manuscript: All authors. \\ Correspondence to: Zhigang Liu. Department of Cardiovascular Surgery, TEDA International Cardiovascular Hospital, 61 Third Avenue, Tianjin, \\ China. Email: c11231348@163.com.
}

Background: Postoperative arrhythmia (POA) is one of the common and serious postoperative complications. This retrospective study was conducted to investigate the clinical factors associated with POA and its short-term prognosis following mitral valve repair.

Methods: A total of 618 patients receiving mitral valve repair between January 2015 and November 2020 in our hospital were included in this retrospective study, including 318 males and 300 females and aged 53.9 \pm 9.3 years. The patients were grouped into arrhythmia and non-arrhythmia groups and investigated for risk factors associated with the prognosis of POA using multivariate logistic regression based on their clinical data. Results: POA was observed in $314(50.8 \%)$ patients and atrial fibrillation (AF) was the most frequent (43.3\%) type of POA. Compared with non-arrhythmia patients, arrhythmia patients had significantly longer time to use vasoactive drug use, longer intensive care unit (ICU) stay and longer hospital stay. In addition, the incidence of postoperative heart failure was significantly higher $(\mathrm{P}<0.05)$. Logistic regression analysis showed that preoperative arrhythmia [odds ratio $(\mathrm{OR})=9.17 ; 95 \%$ confident interval (CI): 4.49-18.10], postoperative pain $(\mathrm{OR}=4.70$; 95\% CI: $1.55-6.12)$ and postoperative hypoxemia $(\mathrm{OR}=3.25$; $95 \%$ CI: $1.04-$ 6.28) were independently associated with POA.

Conclusions: This study demonstrates that the incidence of arrhythmia is relatively high after mitral valve repair and is associated with preoperative arrhythmia, postoperative pain and postoperative hypoxemia.

Keywords: Mitral valve repair; arrhythmia; atrial fibrillation (AF); risk factors; short-term prognosis

Submitted Jun 27, 2021. Accepted for publication Oct 25, 2021.

doi: $10.21037 / \mathrm{cdt}-21-400$

View this article at: https://dx.doi.org/10.21037/cdt-21-400

\section{Introduction}

According to the World Health Organization's (WHO) reports, cardiovascular diseases (CVDs) cause one-third of all annual deaths around the world (1). Cardiac surgeries such as coronary artery bypass grafting (CABG) heart valve replacement are commonly used to treat CVDs to improve their outcomes and to decrease the incidence of mortality. Although the surgery is relatively mature, there are still risks and postoperative complications that need to be addressed (2). Postoperative arrhythmia (POA) is one of the common and serious postoperative complications $(3,4)$, which may result in various impacts on patients, ranging from no clinical symptom to unstable hemodynamics, and complications such as cardiac function failure, renal failure, cerebral infarction and even death (5). The incidence of postoperative supraventricular arrhythmias is reported to be $11-54 \%$, and the incidence of ventricular arrhythmia to be $1.8-13 \%$ following CABG (6). Different 
studies have been performed to improve the management of these complications among the patients undergoing cardiopulmonary surgery $(7,8)$. For example, magnesium sulphate and amiodarone were tested for treating POA that might occur following CABG operation and were found effective to prevent arrhythmia (7). Postoperative atrial fibrillation (POAF) is the most common perioperative cardiac arrhythmia (4). A major risk factor for POAF is advanced age. A study showed that a preoperative left atrial diameter of $\geq 58.0 \mathrm{~mm}$ was a risk factor for atrial fibrillation (AF) recurrence after heart surgery (9). Reliable models for prediction of POA are needed to provide recommendations such as prophylactic treatment of AF for a better outcome in cardiac surgical patients with high risk of developing POA. Three risk models were proposed for preoperative prediction of POAF in cardiac surgical patients: the POAF score, the CHA2DS2-VASC score, and the AF Risk Index. However, they have limited ability to predict POAF occurring in cardiac surgical patients.

A better understanding of what causes the development and progression of arrhythmia in postoperative patients may help reduce the use of pharmacological and electrical procedures aimed at reducing the ventricular rate or ensuring normal sinus rhythm. The aims of this study were to investigate the clinical features of arrhythmia after mitral valve repair and analyze the factors affecting the occurrence and prognosis of arrhythmia. The findings would help reduce the complications and improve the prognosis following valve repair surgery.

We present the following article in accordance with the STROBE reporting checklist (available at https://cdt. amegroups.com/article/view/10.21037/cdt-21-400/rc).

\section{Methods}

\section{Subjects}

This study was a single-center retrospective cohort study. Patients undergoing selective mitral valve repair under cardiopulmonary bypass (CPB) in our hospital between January 2015 and November 2020 were included, irrespective of genders. The patients were included if they were aged 18 to 70 years, had body mass index (BMI) between 15 and $32 \mathrm{~kg} / \mathrm{m}^{2}$, their New York Heart Association (NYHA) classes were II to IV, the American Society of Anesthesiologists (ASA) classes were II-IV. Patients were excluded if they underwent emergency operation, were clinically diagnosed severe coagulation, had severe dysfunction of brain, liver and kidney, endocrine system disease, serious infectious diseases, installed with pacemaker. Patients with incomplete clinical data were also excluded. Written informed consent was obtained from every patient and the study protocols were approved by the Ethics Committee of TEDA International Cardiovascular Hospital (Approval ID: TEDA-C1242). All methods were performed in accordance with the relevant guidelines and regulations and the study was conformed to the provisions of the Declaration of Helsinki (as revised in 2013). Several effects were made to minimize potential bias. Patients treated by the same team of surgeons were enrolled. CPB was preformed following standardized procedure and POA diagnosis was double-confirmed using ECG.

\section{Anesthesia and surgery}

Before surgery, general anesthesia was induced and patients were given tracheal intubation and mechanical ventilation. Intraoperative anesthesia was maintained using combined intravenous and inhalation anesthesia. Myocardium was protected with cardioplegia and hypothermia during the surgery. CPB was performed as previously described (10). Heparin $3 \mathrm{mg} / \mathrm{kg}$ IV was administered before arterial cannulation with a target activated clotting times (ACTs) of more than 480 seconds. During the arterial cannulation, systolic pressure was maintained at $90-100 \mathrm{mmHg}$ and heart rate was maintained at $50-90$ beats $/ \mathrm{min}$. The aortic cannulation was inserted first followed by venous cannulation to establish full CPB. The aorta was clamped and cardioplegia was in intermittent antegrade and retrograde. The leaflet was primarily repaired by resection. The size and shape of resection were determined by the operating surgeon. Ring annuloplasty was performed in case of need. The size of ring was determined based on the area of leaflet of interest using prosthetic aortic valve sizers. Prosthetic ring annuloplasty was performed with a 24-36 mm Carpentier-Edwards Physio ring.

\section{Observation indicators}

Clinical data of the included patients were retrieved from the hospital electronic database, including gender, age and body surface area (BSA), BMI, NYHA class, ASA class, concomitant diseases [pulmonary hypertension, coronary heart disease, arrhythmia, diagnosed previously or by electrocardiography (ECG) after admission]. Preoperative data including preoperative medication and 
echocardiography, intraoperative data such as vital signs, type of operation, number of repaired leaflets, valve adaptation, $\mathrm{CPB}$ and myocardial protection measures, and postoperative data such as arrhythmia, electrolyte acid-base imbalance, hypoxia, hyperthermia, pain, biochemical indicators, and use of vasoactive drugs were collected. Prognosis was measured based on the time of vasoactive drug use, intensive care unit (ICU) stay, hospital stay and other postoperative complications such as heart failure (HF), hypotension, pericardial tamponade, permanent pacemaker implantation and cerebral infarction. All patients were routinely monitored with ECG, blood pressure (BP) and $\mathrm{SpO}_{2}$.

POA was diagnosed with ECG monitoring and confirmed with ECG reexaminations. The POA included AF, atrial premature beat, ventricular premature beat, conduction block, ventricular tachycardia, and sinus bradycardia. Atrial premature beat and ventricular premature beat were frequent premature beat (attack times $>5 / \mathrm{min}$ ). For patients with multiple arrhythmias, the types of arrhythmias were recorded separately but were counted as arrhythmia once.

\section{Statistical analysis}

SPSS 20.0 statistical software was used for statistical analysis. The measurement data of normal distribution were presented as mean \pm standard deviation $(\bar{x} \pm \mathrm{SD})$. The independent sample $t$-test was used for group comparison, and the measurement data of non-normal distribution were expressed as median (m) and interquartile interval (IQR), and compared using the Mann-Whitney $\mathrm{U}$ test. Enumeration data were expressed as number and percentage, and compared using $\chi^{2}$ test or Fisher exact probability test. Variables that had $\mathrm{P}<0.05$ in univariate analysis were included for multiple logistic regression analysis, and the results were presented as odds ratio (OR) and $95 \%$ confident interval (CI). $\mathrm{P}<0.05$ was considered statistically significant. No patient with missing data was included in the analysis.

\section{Results}

A total of 835 patients undergoing selective mitral valve repair under $\mathrm{CPB}$ at our hospital during the study period were eligible for this study. 217 were excluded from the study because they did meet the inclusion criteria $(n=79)$, had other diseases $(n=83)$ or had incomplete clinical data, including loss of follow-up $(\mathrm{n}=55)$ before the analysis. A total of 618 patients were analyzed in this study, including
318 males and 300 females. The average age was 53.9 \pm 9.3 years and BSA was $1.6 \pm 0.2 \mathrm{~m}^{2}$. Preoperative arrhythmia was recorded in 289 patients, 139 and 135 of them had pulmonary hypertension or coronary heart diseases (Table 1).

After mitral valve repair, $372(60.2 \%)$ patients were diagnosed as having arrhythmia. Among them, 234 (62.9\%) patients had preoperative arrhythmia, the rest [138 (37.1\%) cases] occurred as new cases. Two hundred and sixty-four $(40.0 \%)$ cases did not have arrhythmia, although 55 of them had preoperative arrhythmia. In the POA patients, 164 (44.2\%) cases had only one type of arrhythmia, 52 (14.1\%) cases had two types of arrhythmias, $9(2.4 \%)$ had three types of arrhythmias and $7(0.5 \%)$ had four types of arrhythmias. AF was observed in $216(35.0 \%)$ patients and other arrhythmias included atrial premature beat [20 (3.2\%)], ventricular premature beat [18 (2.9\%)], atrioventricular block [44 (7.1\%)], bundle branch block [33 (5.3\%)], and sinus bradycardia [23 (3.7\%)].

In comparison with the non-arrhythmia patients, the arrhythmia patients had significantly older age, higher NYHA and ASA class, more frequent preoperative arrhythmia, more frequent preoperative use of diuretics, larger preoperative size of the left atrium, higher ejection fraction and fractional shortening, longer CPB time, longer aortic cross-clamping time, more surgically repaired leaflets, more postoperative hypokalemia, more hypoxemia, more hyperthermia, more pain, higher postoperative B-type brain natriuretic peptide and lower hemoglobin concentration $(\mathrm{P}<0.05$; Table 1). However, surgical procedures had not significant impact of post-operative arrhythmia.

Logistic regression analysis showed that preoperative arrhythmia (OR $=9.17$; 95\% CI: 4.49-18.10), postoperative pain $(\mathrm{OR}=4.70 ; 95 \% \mathrm{CI}: 1.55-6.12)$ and postoperative hypoxemia $(\mathrm{OR}=3.25 ; 95 \% \mathrm{CI}$ : $1.04-6.28)$ were independently associated with arrhythmias following the surgery (Table 2).

Compared with the non-arrhythmic patients, the arrhythmic patients used vasoactive drugs for longer time (4.4 vs. 2.1 days) and had significantly longer ICU stay (27.9 vs. 21.5 hours) and hospitalization time (17.8 vs. 13.2 days; $\mathrm{P}<0.05$; Table 3). They also had significantly higher heart failure rate (17.7\% vs. $7.6 \%$; Table 3$)$.

\section{Discussion}

Occurrence of POA is influence by a number of factors, such as race, comorbidity, operation methods, monitoring and diagnosis methods. The incidence of POA occurs in 
Table 1 Clinical data of patients with and with arrhythmia after mitral valve repair

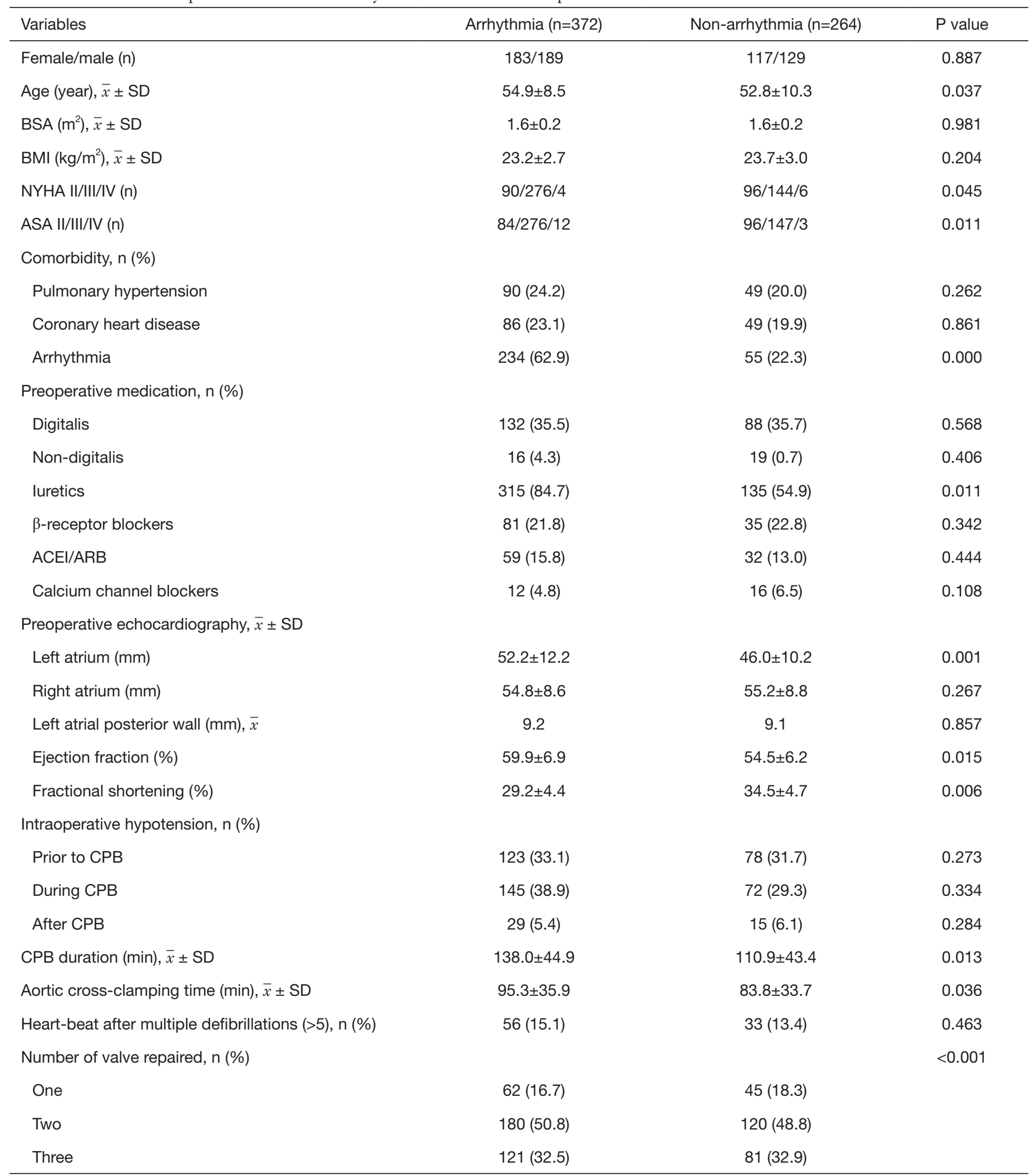

Table 1 (continued) 
Table 1 (continued)

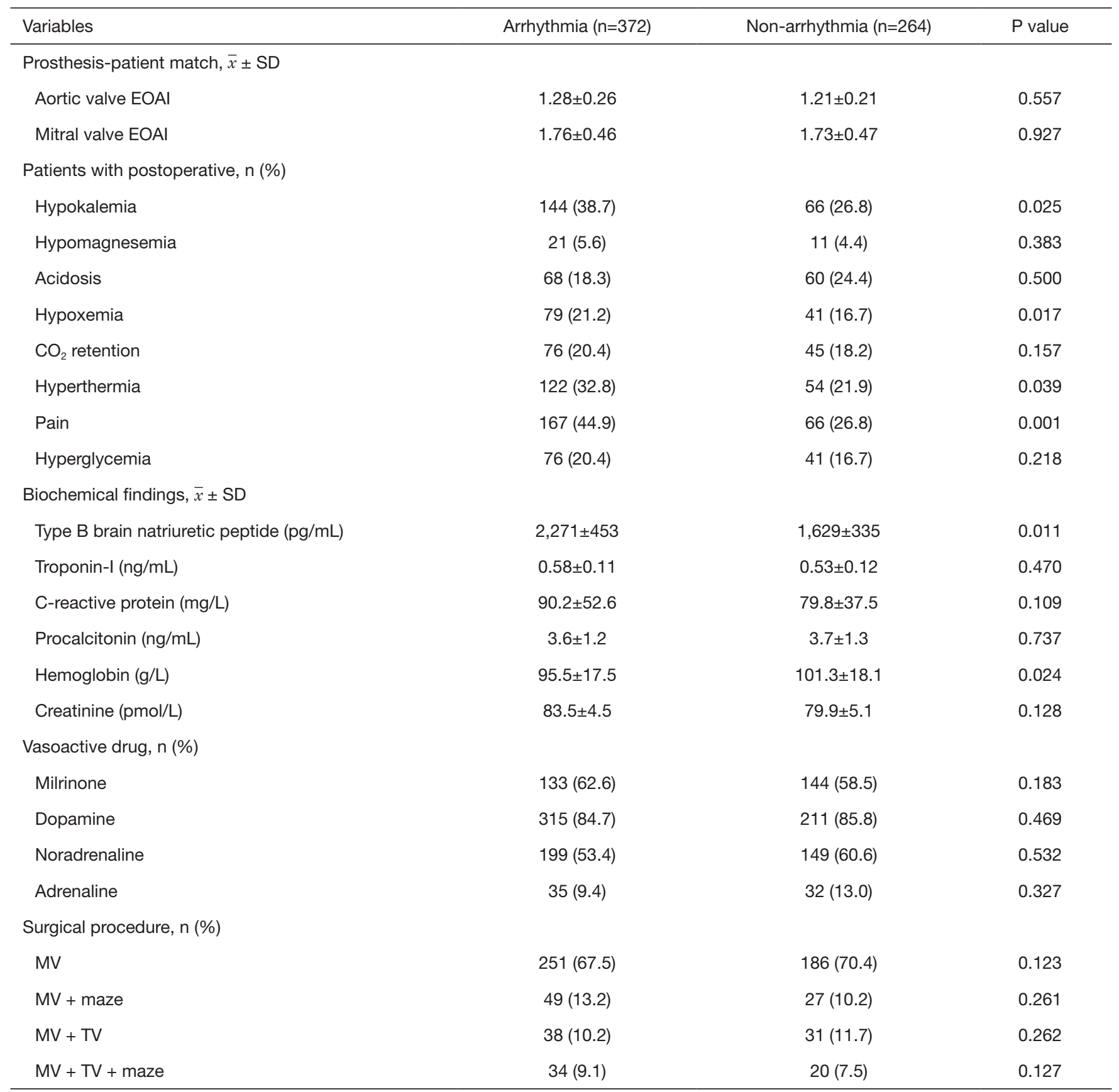

SD, standard deviation; BSA, body surface area; BMI, body mass index; NYHA, New York Heart Association; ASA, American Society of Anesthesiologists; ACEI, angiotensin-converting enzyme inhibitor; ARB, angiotensin II receptor blocker; CPB, cardiopulmonary bypass; EOAI, effective orifice area index; MV, mitral valve; TV, tricuspid valve; AV, aortic valve; maze, the Cox-Maze III procedure.

$10 \%$ to $70 \%$ patients (11). Echahidi et al. showed that the occurrence of $\mathrm{AF}$ is about $30 \%$ after cardiac surgery and about $40 \%$ after valvular surgery (12). In this study, the overall incidence of POA was $60.2 \%$ and the most frequent type was AF (35.0\%), followed by conduction block (5.3\%). $22.3 \%$ of POA were new-onsets.

The occurrence of POA is related to many factors $(13,14)$. In this study, the preoperative, intraoperative and 
Table 2 Risk factors of POA after mitral valve repair

\begin{tabular}{lccccc}
\hline Variables & $\beta$ & Wald & OR & 95\% Cl & P value \\
\hline Preoperative arrhythmia & 0.26 & 16.32 & 9.17 & $4.49-18.10$ & $<0.001$ \\
Postoperative pain & 1.22 & 18.33 & 4.70 & $1.55-6.12$ & $<0.001$ \\
Hypoxemia & 0.667 & 6.34 & 3.25 & $1.04-6.28$ & 0.040 \\
\hline
\end{tabular}

POA, postoperative arrhythmia; OR, odds ratio; $\mathrm{Cl}$, confident interval.

Table 3 Prognosis of patients with and without POA after mitral valve repair

\begin{tabular}{lcc}
\hline Variables & Patients with POA $(\mathrm{n}=372)$ & Patients without POA $(\mathrm{n}=264)$ \\
\hline Usage time of vasoactive drugs $(\mathrm{d}), \bar{x} \pm \mathrm{SD}$ & $4.4 \pm 1.2$ & $2.1 \pm 1.1^{\mathrm{a}}$ \\
ICU stay time $(\mathrm{h}), \bar{x} \pm \mathrm{SD}$ & $27.9 \pm 3.2$ & $21.5 \pm 2.2^{\mathrm{a}}$ \\
Length of hospital stay (d), $\bar{x} \pm \mathrm{SD}$ & $17.8 \pm 4.2$ & $13.2 \pm 4.2^{\mathrm{a}}$ \\
Other postoperative complications, $\mathrm{n}(\%)$ & & $20(7.6)^{\mathrm{a}}$ \\
Heart failure & $66(17.7)$ & $11(13.4)$ \\
Hypotension & $41(11.4)$ & $2(0.8)$ \\
Pericardial tamponade & $2(0.5)$ & $4(2.5)$ \\
Permanent pacemaker implantation & $5(1.3)$ & $4(1.5)$ \\
Delirium & $4(1.1)$ & $1(0.4)$ \\
Cerebral infarction & $3(0.8)$ & $2(0.8)$ \\
Massive pleural effusion (closed-suction drainage) & $2(0.5)$ & $3(1.3)$
\end{tabular}

${ }^{a}, \mathrm{P}<0.05$ vs. patients with POA. POA, postoperative arrhythmia; SD, standard deviation; ICU, intensive care unit.

postoperative factors were analyzed. For preoperative conditions, the history of arrhythmia is the most important factor, which is consistent with previous conclusion that the history of AF increases the risk of POAF (15).

A number of studies have shown that advanced age is a risk factor for POA (14). In our study, compared with nonarrhythmia patients, the average age of patients with POA was significantly greater. However, the age was not found to be associated with POA. This might be attributed to relatively narrow range of ages in the patients included in the study. Race, gender, history of myocardial infarction, obesity, metabolism syndrome, left atrial enlargement ( $>45 \mathrm{~mm}$ ) were previously identified as risk factors for POA (16-18). However, in our analysis, gender, pulmonary hypertension, history of coronary heart disease, BMI and BSA are not related to POA, suggesting that the risk factors for POA may vary among different patient populations.

On other hand, we found that patients with poor cardiac function and high ASA grade and large left atrium have higher risk of POA. In addition to enlarged left atrium, reduced diastolic function of the left ventricle is shown to be a potential risk factor (19).

Almassi et al. found that that the prophylactic use of beta blockers in the perioperative period reduces the incidence of POA (20). However, we did not observe such reduction. The intraoperative risk factors associated with POA include myocardial ischemia and hypoxia, aortic occlusion and atrial damage (21) and hemodynamic fluctuation (22). In our multivariate analysis, although none of these variables is identified to be associated with POA, POA patients appear to have longer $\mathrm{CPB}$ and longer aortic clamping time with more leaflets repaired or replaced in the surgery.

Cardiac surgery often causes vasospasm, systemic inflammation, excessive release of catecholamine, changes in the sympathetic and parasympathetic nerves, and the activation of neurohumoral systems (23). These 
are potential factors that lead to POA (12). Systemic inflammatory response generates oxidative tissue damage through the release of oxygen free radicals, which reduce effective refractory period that generates action potential to induce arrhythmia (24-26). A number of studies have shown that the new onset of AF is related to the inflammatory response with high $\mathrm{C}$-reactive protein concentration $(27,28)$. In our study, the level C-reactive protein is higher in POA patients but not statistically different between the two groups, suggesting that it is still important to play attention to the inflammation in the perioperative period.

The main postoperative independent risk factors associated with POA are pain and hypoxemia. Pain may stimulate the sympathetic nerves, leading to ectopic atrial rhythms and triggering arrhythmia $(29,30)$. Hooten et al. found that sufficient postoperative analgesia can effectively reduce POA (31). Pain can be managed via blocking the paravertebral nerves during the operation, use of analgesia pump, or intermittent use of analgesics after the operation. Hypovolemia, anemia, hypoxia and carbon dioxide retention can over-activate the sympathetic nervous system, leading to massive release of endogenous catecholamine and arrhythmia (32). Our results also showed that hypoxemia has a significant effect on POA. Electrolyte disorders can cause abnormal activation of cardiac myocytes, increase the sensitivity of the heart muscle and generate arrhythmia (20). In addition, Hernández-Leiva et al. showed that high preoperative B-type natriuretic peptide is an indicator of poor postoperative prognosis (33), which is consistent with our results, although we analyzed the postoperative level of B-type natriuretic peptide.

A large number of studies have shown that POA is associated with acute renal damage, risk of heart failure and stroke, ICU stay and hospital time (34), long-term mortality (35), and patient's treatment cost. This study also showed that POA patients were associated with longer duration of vasoactive drugs use, longer ICU stay and longer hospital stay. They also had higher postoperative heart failure. Although the incidence of stroke in this study is not associated with POA, it is still higher in POA than non-POA patients. This disassociation might be due to relatively short observation period for postoperative complications in this study.

There are some limitations in the study. As a single center and retrospective study, the sample size was relatively small, the study population was highly selected and had a narrow range of age. The follow-up time was relatively short and important variables such as mortality were unable to be included for analysis. The conclusions of this study, therefore, need to be validated with large perspective multiple-center studies.

\section{Conclusions}

Our study demonstrates that the incidence of arrhythmia is relatively high after mitral valve repair and the incidence of POA is associated with preoperative arrhythmia, postoperative pain and postoperative hypoxemia. Furthermore, arrhythmia patients also had longer vasoactive drug use, longer ICU stay, longer hospitalization time and higher heart failure rate.

\section{Acknowledgments}

We would like to thank Professor Illimar Altosaar, University of Ottawa, Canada, for editing the language of this manuscript.

Funding: None.

\section{Footnote}

Reporting Checklist: The authors have completed the STROBE reporting checklist. Available at https://cdt. amegroups.com/article/view/10.21037/cdt-21-400/rc

Data Sharing Statement: Available at https://cdt.amegroups. com/article/view/10.21037/cdt-21-400/dss

Conflicts of Interest: All authors have completed the ICMJE uniform disclosure form (available at https://cdt.amegroups. com/article/view/10.21037/cdt-21-400/coif). The authors have no conflicts of interests to declare.

Ethical Statement: The authors are accountable for all aspects of the work in ensuring that questions related to the accuracy or integrity of any part of the work are appropriately investigated and resolved. Informed consent was obtained from every patient, and the study protocols were approved by the Ethics Committee of TEDA International Cardiovascular Hospital (Approval ID: TEDA-C1242). All methods were performed in accordance with the relevant guidelines and regulations and the study was conformed to the provisions of the Declaration of Helsinki (as revised in 2013).

Open Access Statement: This is an Open Access article 
distributed in accordance with the Creative Commons Attribution-NonCommercial-NoDerivs 4.0 International License (CC BY-NC-ND 4.0), which permits the noncommercial replication and distribution of the article with the strict proviso that no changes or edits are made and the original work is properly cited (including links to both the formal publication through the relevant DOI and the license). See: https://creativecommons.org/licenses/by-nc-nd/4.0/.

\section{References}

1. Firoozabadi MD, Sheikhi MA, Rahmani H, et al. Risks of on-pump coronary artery bypass grafting surgery in patients with chronic obstructive pulmonary disease due to sulfur mustard. Postepy Dermatol Alergol 2017;34:429-32.

2. Stephens RS, Whitman GJ. Postoperative Critical Care of the Adult Cardiac Surgical Patient: Part II: Procedure-Specific Considerations, Management of Complications, and Quality Improvement. Crit Care Med 2015;43:1995-2014.

3. Khan J, Khan N, Loisa E, et al. Increasing Occurrence of Postoperative Atrial Fibrillation in Contemporary Cardiac Surgery. J Cardiothorac Vasc Anesth 2016;30:1302-7.

4. Bessissow A, Khan J, Devereaux PJ, et al. Postoperative atrial fibrillation in non-cardiac and cardiac surgery: an overview. J Thromb Haemost 2015;13 Suppl 1:S304-12.

5. Koletsis EN, Prokakis C, Crockett JR, et al. Prognostic factors of atrial fibrillation following elective coronary artery bypass grafting: the impact of quantified intraoperative myocardial ischemia. J Cardiothorac Surg 2011;6:127.

6. Banach M, Rysz J, Drozdz JA, et al. Risk factors of atrial fibrillation following coronary artery bypass grafting: a preliminary report. Circ J 2006;70:438-41.

7. Tiryakioglu O, Demirtas S, Ari H, et al. Magnesium sulphate and amiodarone prophylaxis for prevention of postoperative arrhythmia in coronary by-pass operations. J Cardiothorac Surg 2009;4:8.

8. Lan YT, Lee JC, Wetzel G. Postoperative arrhythmia. Curr Opin Cardiol 2003;18:73-8.

9. Ishii Y, Sakamoto SI, Miyagi Y, et al. Risk Factors of Recurrence of Atrial Fibrillation (AF) After AF Surgery in Patients With AF and Mitral Valve Disease. Semin Thorac Cardiovasc Surg 2018;30:271-8.

10. Sarkar M, Prabhu V. Basics of cardiopulmonary bypass. Indian J Anaesth 2017;61:760-7.

11. Todorov H, Janssen I, Honndorf S, et al. Clinical significance and risk factors for new onset and recurring atrial fibrillation following cardiac surgery - a retrospective data analysis. BMC Anesthesiol 2017;17:163.

12. Echahidi N, Pibarot P, O'Hara G, et al. Mechanisms, prevention, and treatment of atrial fibrillation after cardiac surgery. J Am Coll Cardiol 2008;51:793-801.

13. Jaimes MC, Torrado LAA, Reyes NFS, et al. Hypothyroidism is a Risk Factor for Atrial Fibrillation after Coronary Artery Bypass Graft. Braz J Cardiovasc Surg 2017;32:475-80.

14. Kavurmaci O, Akcam TI, Ergonul AG, et al. Is the Risk of Postoperative Atrial Fibrillation Predictable in Patients Undergoing Surgery Due to Primary Lung Cancer? Heart Lung Circ 2018;27:835-41.

15. Mathew JP, Fontes ML, Tudor IC, et al. A multicenter risk index for atrial fibrillation after cardiac surgery. JAMA 2004;291:1720-9.

16. Guenancia C, Pujos C, Debomy F, et al. Incidence and Predictors of New-Onset Silent Atrial Fibrillation after Coronary Artery Bypass Graft Surgery. Biomed Res Int 2015;2015:703685.

17. Phan K, Khuong JN, Xu J, et al. Obesity and postoperative atrial fibrillation in patients undergoing cardiac surgery: Systematic review and meta-analysis. Int J Cardiol 2016;217:49-57.

18. Vural Ü, Ağlar AA. What is the role of metabolic syndrome and obesity for postoperative atrial fibrillation after coronary bypass grafting? BMC Cardiovasc Disord 2019;19:147.

19. Haghjoo M, Basiri H, Salek M, et al. Predictors of postoperative atrial fibrillation after coronary artery bypass graft surgery. Indian Pacing Electrophysiol J 2008;8:94-101.

20. Almassi GH, Pecsi SA, Collins JF, et al. Predictors and impact of postoperative atrial fibrillation on patients' outcomes: a report from the Randomized On Versus Off Bypass trial. J Thorac Cardiovasc Surg 2012;143:93-102.

21. Maisel WH, Rawn JD, Stevenson WG. Atrial fibrillation after cardiac surgery. Ann Intern Med 2001;135:1061-73.

22. Dobrev D, Aguilar M, Heijman J, et al. Postoperative atrial fibrillation: mechanisms, manifestations and management. Nat Rev Cardiol 2019;16:417-36.

23. Rouleau JL, Moyé LA, de Champlain J, et al. Activation of neurohumoral systems following acute myocardial infarction. Am J Cardiol 1991;68:80D-6D.

24. Zakkar M, Ascione R, James AF, et al. Inflammation, oxidative stress and postoperative atrial fibrillation in cardiac surgery. Pharmacol Ther 2015;154:13-20.

25. Korantzopoulos P, Letsas K, Fragakis N, et al. Oxidative 
stress and atrial fibrillation: an update. Free Radic Res 2018;52:1199-209.

26. Elahi MM, Flatman S, Matata BM. Tracing the origins of postoperative atrial fibrillation: the concept of oxidative stress-mediated myocardial injury phenomenon. Eur J Cardiovasc Prev Rehabil 2008;15:735-41.

27. Li T, Sun ZL, Xie QY. Meta-analysis Identifies Serum C-Reactive Protein as an Indicator of Atrial Fibrillation Risk After Coronary Artery Bypass Graft. Am J Ther 2016;23:e1586-96.

28. Jakubová M, Mitro P, Stančák B, et al. The occurrence of postoperative atrial fibrillation according to different surgical settings in cardiac surgery patients. Interact Cardiovasc Thorac Surg 2012;15:1007-12.

29. Jiang Z, Dai JQ, Shi C, et al. Influence of patientcontrolled i.v. analgesia with opioids on supraventricular arrhythmias after pulmonary resection. Br J Anaesth 2009; 103:364-8.

30. de Oliveira RM, Tenório SB, Tanaka PP, et al. Control of pain through epidural block and incidence of cardiac dysrhythmias in postoperative period of thoracic and major abdominal surgical procedures: a comparative study. Rev
Bras Anestesiol 2012;62:10-8.

31. Hooten WM, Karanikolas M, Swarm R, et al. Postoperative pain management following bilateral lung volume reduction surgery for severe emphysema. Anaesth Intensive Care 2005;33:591-6.

32. Christians KK, Wu B, Quebbeman EJ, et al. Postoperative atrial fibrillation in noncardiothoracic surgical patients. Am J Surg 2001;182:713-5.

33. Hernández-Leiva E, Dennis R, Isaza D, et al. Hemoglobin and B-type natriuretic peptide preoperative values but not inflammatory markers, are associated with postoperative morbidity in cardiac surgery: a prospective cohort analytic study. J Cardiothorac Surg 2013;8:170.

34. Mariscalco G, Biancari F, Zanobini M, et al. Bedside tool for predicting the risk of postoperative atrial fibrillation after cardiac surgery: the POAF score. J Am Heart Assoc 2014;3:e000752.

35. Mariscalco G, Engström KG. Postoperative atrial fibrillation is associated with late mortality after coronary surgery, but not after valvular surgery. Ann Thorac Surg 2009;88:1871-6.
Cite this article as: Zhu Y, Jing W, Lv P, Zhu Y, Liu Z. Clinical factors associated with arrhythmia and short-term prognosis following mitral valve repair: a retrospective cohort study. Cardiovasc Diagn Ther 2022;12(1):114-122. doi: 10.21037/cdt-21-400 